\title{
Análisis clausular de dos tipos de narraciones escritas por estudiantes de educación básica ${ }^{1}$
}

\author{
Clause analysis in two narrative types written by elementary school students
}

\author{
Manuel Rubio2
}

\section{Resumen}

El propósito de este artículo es comparar tanto el tipo de cláusulas en textos narrativos escritos producidos por estudiantes de tres niveles de enseñanza básica $\left(3^{\circ}, 5^{\circ}\right.$ y $\left.7^{\circ}\right)$ como el grado de dependencia y relaciones lógico-semánticas en los complejos clausulares, con la finalidad de establecer los principales cambios que es posible observar a medida que se avanza en escolaridad. El corpus está constituido por 180 textos producidos por estudiantes de $3^{\circ}, 5^{\circ}$ y $7^{\circ}$ básico bajo dos condiciones de escritura. Los resultados indican el predominio significativo de los complejos clausulares por sobre las cláusulas simples en todos los niveles, con dependencia paratáctica y relaciones lógico-semánticas de expansión mediante la especificación de elementos circunstanciales y agregación de información nueva usando elementos conjuntivos. No obstante, se observa un patrón de incremento de las relaciones hipotácticas, que logra niveles significativos al comparar los textos de los estudiantes de $5^{\circ}$ con los de $3^{\circ}$ básico. Se concluye que los estudiantes progresivamente avanzan en la condensación de información en una misma estructura sintagmática, produciéndose un cambio cualitativo entre el $3^{\circ}$ y el $5^{\circ}$ básico. En general, los estudiantes escriben narraciones que relevan las acciones como eventos observables, cuyos participantes prácticamente no hablan ni reflexionan sobre su quehacer. Sin embargo, hay una tendencia incipiente a enriquecer sus producciones incorporando estos aspectos a medida que se avanza en la escolaridad.

Palabras clave: complejos clausulares, producción escrita, desarrollo sintáctico.

\begin{abstract}
This article aims to compare both the type of clauses in narratives written by elementary school students and the degree of interdependency (parataxis and hypotaxis) and logico-semantic relations of clause complexes to identify the main changes observed as schooling progresses. The corpus consists of 180 texts produced by students of $3^{\text {rd }}, 5^{\text {th }}$ and $7^{\text {th }}$ elementary levels under two writing conditions. Results indicate a significant predominance of clause complexes in the three elementary levels, with paratactic interdependency and logico-semantic relations of expansion conveyed by some circumstantial features and new information added through conjunctions. Nevertheless, an increased hypotactic relation pattern is observed. This pattern reaches significant levels when comparing texts written by $5^{\text {th }}$ grade to $3^{\text {rd }}$ grade students. It is concluded that students progressively advance by condensing information in the same syntagmatic structure. This means a qualitative change between $3^{\text {rd }}$ and $5^{\text {th }}$ elementary levels. In general, students write narratives which present actions as observable events, whose participants virtually do not talk neither reflect on their actions. However, there is an emerging trend to enrich their productions incorporating these aspects as they advance in schooling.
\end{abstract}

Key words: clause complexes, written production, syntactic development.

\footnotetext{
${ }^{1}$ Este artículo se realizó en el marco del proyecto Fondecyt/Iniciación 11130010.

${ }^{2}$ Profesor Asociado, Universidad de Santiago de Chile. https://orcid.org/0000-0003-2314-1781 manuel.rubio@usach.cl
}

Recibido: 14.07.2020

Aceptado: 01.12.2020 


\section{Introducción}

En contraste con la oralidad, la producción escrita es una práctica de construcción de significados condicionada cognitiva y culturalmente que requiere de instrucción sistemática. Junto con el conocimiento de la lengua, la configuración de un texto coherente y efectivo supone un proceso de razonamiento para activar conocimiento temático y estructuras discursivas, cuya articulación supone la problematización de la situación comunicativa y de los efectos deseados en los eventuales lectores. En efecto, escribir es poner en práctica un proceso de reflexión para resolver problemas relativos a qué decir para lograr ciertos propósitos y a cómo hacerlo de manera eficaz, oportuna y adecuada (Beard, Myhill, Riley y Nystrand, 2009; Grabe y Kaplan, 2014; Kellog, 2008; Pinheiro y De Freitas Rossi, 2008).

Según Kellog (2008), llegar a ser un escritor experto implica unas dos décadas de maduración, instrucción y práctica. En una etapa inicial, correspondiente a la escolaridad obligatoria, los estudiantes tienden a centrarse en sí mismos y en el despliegue de recursos cognitivos orientados a la recuperación de ideas, por lo cual tanto los procesos de planificación, redacción y revisión tienden a ser limitados. Desde esta perspectiva, un primer desafío en la enseñanza de la producción escrita es facilitar el enriquecimiento de dichos procesos y la interacción entre ellos conducente a la toma de conciencia sobre las representaciones que el autor tiene de sí mismo y del texto producido.

No obstante, pareciera ser que el andamiaje para el modelaje y una práctica deliberada orientada al desarrollo de la producción escrita no sería una prioridad en el sistema escolar (Meneses, 2006; 2008). Hay dos factores que pudieran explicar dicha situación: por un lado, la falta de preparación de los profesores y, por otro, la carencia de materiales didácticos adecuados (Koster, Tribushinina, de Jong y van den Bergh, 2015). Al respecto, diversos investigadores (Schleppegrel, 2004; Rose y Acevedo, 2017) destacan que la labor de modelaje de la escritura por parte del docente requiere que este se enfoque en los rasgos lingüísticos que caracterizan los géneros discursivos que debe enseñar. En consecuencia, el docente debiera tener conocimiento para develar los propósitos comunicativos que realizan las opciones léxicogramaticales plasmadas en un determinado texto escrito.

En relación con los textos escritos por escolares básicos, algunos estudios los caracterizan como relativamente deficientes, puesto que son pocos los estudiantes que logran que sus textos se atengan al tema y sean adecuados a la situación propuesta, y, por el contrario, despliegan una reducida variedad y adecuación en el uso de recursos cohesivos y de estructuración textual (Mineduc 2009, 2015; Marinkovich, 2006; Sotomayor, Lucchini, Bedwell, Biedma, Hernández y Molina, 2013). Benitez (2009), al analizar escritos de escolares de $3^{\circ}$ básico, concluye que ninguno alcanza los niveles de respuestas "idóneo" y "excelente", puesto que en sus escritos se constatan saltos en la secuencia temporal de los eventos narrados, prácticamente no se utiliza la subordinación y la coordinación está limitada por el uso de la conjunción "y".

Paralelamente, los estudios sobre el desempeño escrito de los escolares desde una perspectiva lingüística son escasos (Christie y Derewianka, 2010; Koster, Tribushinina, de Jong y van den Bergh, 2015; Rubio, 2019) y diversos investigadores del ámbito hispanohablante indican que tales estudios se han realizado en lengua inglesa (Coloma, Peñaloza, Fernández, 2007; Ow y Alvarado, 2013). Concretamente en Chile, Bañales, Ahumada, Martínez, Martínez y Messina (2018) efectuaron una revisión de las investigaciones sobre la producción escrita desarrollada en la Educación Básica. De los 27 estudios identificados, solo tres de ellos se enfocan en aspectos sintácticos: dos sobre complejidad sintáctica y uno sobre recursos de coherencia local. Desde el 


\section{Manuel Rubio}

2016 al 2019, se publicaron tres artículos enfocados en aspectos sintáctico en la escritura de escolares de Educación Básica. Aravena y Hugo (2016) analizan el desarrollo de la complejidad sintáctica de escolares básicos en dos modalidades textuales: narración y explicación, concluyendo que esta se correlaciona con la cultura letrada de la familia y la edad de los sujetos. Rubio (2019) analiza los verbos y los grupos verbales en la configuración de la experiencia, constatando una orientación progresiva, a medida que se avanza en la escolaridad, desde la representación externa de los eventos a una orientación más interna. Díaz Oyarce (2019) se centra en los elementos de conexión en textos narrativos de estudiantes de $4^{\circ}, 5^{\circ}$ y $6^{\circ}$ básicos, los cuales se incrementan a medida que se avanza en la escolaridad.

En otras palabras, ante la falta de investigaciones sobre la producción escrita de los escolares desde una perspectiva lingüística, es necesario indagar tal desempeño en los textos escritos por escolares de Educación Básica en Chile. Al respecto, el análisis de las cláusulas que componen dichos textos puede ser un indicador para identificar posibles cambios a medida que se avanza en la escolaridad. En efecto, los recursos gramaticales empleados dan cuenta de la capacidad de un escritor en formación para construir significados y plasmarlos estratégicamente en el texto producido. Por tanto, su análisis y posibilidades de ampliación y enriquecimiento contribuyen a abordar el rol de la sintaxis en la producción textual, particularmente en lo referido a la densidad y conexión informativa.

La hipótesis de trabajo es que la escolaridad impacta en el incremento de complejos clausulares presentes en los textos escritos por los escolares, pues al vincular dos o más cláusulas, el escritor establece una relación semántica de los eventos representados por ellas, construyendo el flujo de dichos eventos como un todo, una secuencia que supone una relación más estricta y explícita del significado que se desea compartir (Halliday y Mathiessen, 2004; Thompson, 2014; Eggins, 2013).

El propósito del artículo es comparar el tipo de cláusulas presente en los textos narrativos producidos por estudiantes de tres niveles de enseñanza básica $\left(3^{\circ}, 5^{\circ}\right.$ y $\left.7^{\circ}\right)$, así como el grado de dependencia y relaciones lógico-semánticas en los complejos clausulares, con la finalidad de establecer los principales cambios que es posible observar a medida que se avanza en la escolaridad. Se eligió la narración, debido a que es una modalidad textual cuyo dominio se adquiere a temprana edad e implica el uso de recursos lingüísticos para relacionar eventos y presentarlos cohesivamente (Aravena, 2011; Christie y Derewianka, 2010; Tomasello, 2003). Además, el texto narrativo permite observar el despliegue de elementos subjetivos que el narrador le asigna a los actantes en el relato, explicando de este modo las causas que motivan su acción, lo cual es denominado como paisajes de la conciencia (Bocaz, 1996).

\section{Marco teórico}

\section{Cláusulas y complejos clausulares}

En la perspectiva funcionalista propuesta por Halliday, la lengua se concibe como semiótica social, es decir, como un recurso para la construcción de significados mediante una serie de sistemas de opciones. En este sentido, un texto es el producto de las elecciones de un hablante en una situación social dada, en el cual se realizan sus opciones en una serie de patrones paradigmáticos disponibles para la realización de tres tipos de significados: la representación de la experiencia, el intercambio entre los interlocutores y la organización del mensaje. En esta línea, la selección léxica y su correspondiente configuración sintagmática se enfocan desde el nivel semántico. Dentro de dicha 


\section{Manuel Rubio}

configuración, cada uno de sus componentes se describen en términos de sus interrelaciones funcionales (Halliday y Mathiessen, 1999, 2004; Thompson, 2014, Eggins, 2013; Gutiérrez, 2008).

De acuerdo con lo planteado por la lingüística sistémico funcional, se conceptualiza la cláusula como una configuración sintagmática que gira en torno a un centro nuclear realizado por un verbo o grupo verbal, cuya función es producir significados. En la cláusula se concretan las elecciones de los hablantes para representar y comunicar la experiencia, establecer relaciones lógicas entre los eventos, intercambiar con otros y organizar el mensaje (Halliday y Mathiessen, 2004; Thompson, 2014, Eggins, 2013, Gutiérrez 2008). Un complejo clausular es una unidad que conecta dos o más cláusulas y, según el tipo de relación establecido entre ellas, este puede analizarse desde dos perspectivas: el grado de interdependencia (la vinculación entre cláusulas según su estatus) y la relación lógica semántica (similitud o diferencia en el orden de experiencia representado por las cláusulas relacionadas).

Congruentemente, la cláusula se concibe como una estructura sintáctica, cuyo núcleo constituyente es un proceso representado por un verbo o un grupo verbal. Dicha configuración realiza una función semiótica articulando una serie de opciones para construir e intercambiar la experiencia. Prototípicamente, el proceso está circunscrito temporal y modalmente mediante la conjugación verbal. No obstante, el proceso puede concretarse a través de una forma nominal del verbo, es decir, sin finitud temporal y modal, y en una posición sintáctica dependiente de un proceso principal (Halliday y Mathiessen, 1999, 2004; Thompson, 2014; Eggins, 2013; Gutiérrez, 2008). Veamos algunos ejemplos extractados del corpus de análisis.

1) "Mi hermana nació en una clínica" (E20-3ํásico).

2) "En mis vacasiones siempre quise conocer el norte" (E3-5 básico).

3) "Cuando de repente caminando encuentran un túnel" (E8 $-5^{\circ}$ básico).

En 1) el núcleo de la cláusula (nació) representa un proceso observable (proceso material) ocurrido en el pasado el cual se concibe como un intercambio de información con un alto nivel de certeza. En 2) el núcleo está constituido por un grupo verbal (quise conocer), una unidad de significado que, como conjunto, representa un proceso no observable (proceso mental) en el pasado, el cual se configura como un intercambio de información sobre un hecho deseado, una posibilidad. En 3) encontramos una cláusula con dos verbos, uno conjugado que representa el proceso principal (encontrar) que se sitúa como algo observable, externo al sujeto (proceso material) y un verbo no conjugado (caminando) que representa un proceso dependiente del primero, como una expansión de tipo circunstancial.

En torno al núcleo de la cláusula, se establecen una serie de posiciones funcionales que determinan las cualidades estructurales de las palabras o grupos de palabras que deben ser seleccionadas para llenar dicha posición. De este modo, se construye e intercambia la experiencia, se conectan lógicamente los eventos, se asume un rol discursivo y se organiza el mensaje. En otras palabras, la cláusula es una estructura multidimensional, en la cual el hablante concreta diferentes opciones de significación y las estructura sintagmáticamente. En efecto, en la cláusula se realizan tres configuraciones de significados distintos y simultáneos, como se observa en el ejemplo 4) extractado del corpus de análisis.

4) "Mi papá me llevaria a su departamento por el fin de semana, para estar con él y mi hermano" (E1 - $7^{\circ}$ básico) 
Manuel Rubio

\begin{tabular}{|c|c|c|c|c|c|c|}
\hline $\begin{array}{l}\text { Estructura } \\
\text { funcional }\end{array}$ & \multicolumn{6}{|c|}{ Funciones } \\
\hline Transitividad & $\begin{array}{l}\text { Actor: } \quad \mathrm{mi} \\
\text { papá }\end{array}$ & $\begin{array}{l}\text { Meta: } \\
\text { me }\end{array}$ & $\begin{array}{l}\text { Proceso } \\
\text { material: } \\
\text { llevaría }\end{array}$ & \multicolumn{3}{|c|}{$\begin{array}{l}\text { Circunstancia } \\
\text { a) lugar: a su departamento } \\
\text { b) duración: por el fin de semana } \\
\text { c) finalidad: para estar con él mi } \\
\text { hermano }\end{array}$} \\
\hline \multirow[t]{2}{*}{$\begin{array}{l}\text { Interpersonal } \\
\text { (intercambio) }\end{array}$} & \multicolumn{3}{|c|}{$\begin{array}{l}\text { Núcleo modal: } \\
\text { mi papá + morfema ía }\end{array}$} & \multicolumn{3}{|c|}{$\begin{array}{l}\text { Residuo: llevar a su departamento por el fin } \\
\text { de semana para estar con él y mi hermano }\end{array}$} \\
\hline & $\begin{array}{l}\text { Sujeto } \\
\text { lexicalizado: } \\
\text { mi papá } \\
\text { Sujeto } \\
\text { gramatica- } \\
\text { lizado: } \\
\text { morfema ía }\end{array}$ & \multicolumn{2}{|c|}{$\begin{array}{l}\text { Finito temporal y } \\
\text { modal: } \\
\text { morfema ía }\end{array}$} & $\begin{array}{c}\text { Predicador: } \\
\text { llevar }\end{array}$ & $\begin{array}{l}\text { Comple- } \\
\text { mento: } \\
\text { me }\end{array}$ & $\begin{array}{l}\text { Adjuntos: } \\
\begin{array}{ll}\text { - } & \text { a su depto. } \\
\text { - } & \text { por el fin de } \\
& \text { semana } \\
\text { - } & \text { para estar con } \\
\text { él y mi } \\
\text { hermano }\end{array}\end{array}$ \\
\hline Textual & \multicolumn{4}{|c|}{ Tema: mi papá me } & \multicolumn{2}{|c|}{$\begin{array}{l}\text { Rema: llevaría a su } \\
\text { departamento por el fin de } \\
\text { semana para estar con él y mi } \\
\text { hermano }\end{array}$} \\
\hline
\end{tabular}

Estructuralmente, como se observa en el análisis de 4), la cláusula es una unidad cuyas partes tienen una función distintiva en el sistema de transitividad (actor, proceso, meta y circunstancias), de intercambio (el núcleo modal compuesto por los significados del sujeto y el operador finito; y el residuo constituido por predicador, complemento y adjunto) y textual (tipos de temas, rema) (Halliday y Mathiessen, 2004; Thompson, 2014; Eggins, 2013; Gutiérrez, 2008).

Dos o más cláusulas pueden vincularse entre sí conformando complejos clausulares, es decir, donde se conectan procesos de acuerdo con a) grados de interdependencia de las cláusulas entre sí y b) relaciones lógico-semánticas. En otras palabras, un complejo clausular integra una secuencia de eventos encadenados por vínculos que establecen una jerarquía entre los eventos representados y una conexión semántica entre ellas (Thompson, 2014; Martin, Mathiessen \& Painter, 1997; Halliday y Mathiessen, 1999, 2004; Eggins, 2013; Rose y Martin, 2012). Por ejemplo,

5) "Nos vajamos del bus y caminamos hasta donde ivamos a quedarnos" (E2 -7º básico).

6) Un dia los padres de Belinda decidieron cambiarla a otro colegio en el cual se enamoro de un niño llamado David del cual se enamoro profundamente (E5 -5º́sico).

Al analizar los complejos clausulares 5) y 6) de acuerdo con el grado de interdependencia, es decir, las opciones disponibles para conectar los procesos representados según su estatus, podemos constatar que la relación entre las cláusulas que los componen es diferente en ambos casos. En 5), la relación de los procesos materiales "bajamos" y "caminamos" es de igualdad y se reúnen por un elemento cojuntivo ("y"), de tal modo que la primera cláusula inicia el proceso y la 


\section{Manuel Rubio}

otra lo continúa. A este tipo de relación se le denomina paratáctica. En la parataxis, cada una de las cláusulas puede funcionar como un todo independiente.

En cambio, si la relación es asimétrica, en que una de ellas tiene una posición dominante y la otra es dependiente, la relación es hipotáctica. En la hipotaxis, la cláusula dominante es libre, pero no la dependiente. En el caso 5), el tercer proceso ("íbamos a quedarnos") forma parte de una circunstancia del proceso dominante ("caminamos"), esto es, está en una relación de dependencia de dicho proceso. En el ejemplo 6), existe una cláusula cuyo proceso es dominante ("decidieron cambiar") seguido de tres cláusulas que expresan procesos dependientes ("se enamoró", "llamado", "se enamoró"), o sea, hay una relación de asimetría, la cual se denomina hipotaxis.

Como se observa en 5), en un texto es posible configurar complejos clausulares que combinen tanto relaciones paratácticas como hipotácticas.

Respecto de las relaciones lógico-semánticas, las elecciones posibles son la proyección y la expansión. En la proyección, una cláusula principal (ya sea iniciadora o dominante) es seguida por otra (continuadora o dependiente) esbozando ideas o locuciones; es decir, en el primer caso se representa el pensamiento de un participante y, en el segundo, el discurso ya sea por procedimientos directos o indirectos.

7) "Mi papá Danilo y yo nunca la abiamos visto y estabamos nerviosos porque creiamos que nos iba a echar de la casa" (E13- $7^{\circ}$ básico).

8) "Io le dije la verdad que io fui al concierto de Justin Bieber y estuve en la primera fila" (E3 - $7^{\circ}$ básico).

9) "Cuando nos despedimos él nos dijo "espero verlos el otro año" (E11 - $7^{\circ}$ básico).

En 7) el verbo creer proyecta una idea ("nos iba a echar de la casa"), algo que se representa en el texto como localizado en la mente de las personas y no expresado verbalmente. Por el contrario, en 8) y 9) se consigna en el texto el discurso directo de una persona representada en el discurso, en ambos casos introducidos por el verbo decir. Cabe hacer notar, sin embargo, que en el corpus de análisis, como se verá en los resultados, el porcentaje de complejos clausulares con relaciones lógico-semánticas de proyección es escaso en los textos de los escolares. De este bajo porcentaje, las proyecciones de ideas son proporcionalmente mucho menor que las de locución.

Respecto de las relaciones lógico-semánticas de expansión, esta se realiza mediante diferentes procedimientos para desarrollar la información presente en la cláusula iniciadora $\mathrm{o}$ domintante, usando procedimientos tales como la reformulación, la especificación, la ejemplificación, la explicitación de excepciones o la descripción de rasgos circunstanciales. Algunos ejemplos extractados del corpus de análisis son:

10) "Estas vacaciones fueron mis mejores vacaciones fueron alegres, tristes, chistosas" (E13 - $7^{\circ}$ básico).

11) "El día de devolvernos pasamos por Villarrica y pasamos a unas tinajas (eran como $\underline{\text { llacucis }}) "$ (E16 - $7^{\circ}$ básico).

12) "Laura empezó a desesperarse porque no encontraba a diego" (E15 - 7 básico).

13) "Cristoval y Cote se conocieron cuando Cristoval atrabeso el tunel”" (E18 - $7^{\circ}$ básico).

En 10), se expande la cláusula principal (“estas fueron mis mejores vacaciones”) a través de la descripción con calificativos. En 11), se expande un elemento de la segunda cláusula ("y pasamos a unas tinajas") especificando cómo eran las tinajas mediante un procedimiento de

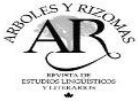




\section{Manuel Rubio}

comparación. En 12), se expande la cláusula dominante ("Laura empezó a desesperarse") mediante un rasgo circunstancial de causa. En 13) la cláusula dominante ("Cristoval y Cote se conocieron") se expande mediante un rasgo circustancial de tiempo.

\section{El desarrollo sintáctico}

Desde el punto de vista ontogenético, el desarrollo de la lengua es un proceso condicionado culturalmente, manifestado en una creciente cantidad de recursos disponibles para producir y compartir significados. En consecuencia, la configuración de un texto implica la concreción de opciones de significado disponibles en el sistema de la lengua, concebido como una red de sistemas de opciones.

La interrelación de una serie de procesos en una secuencia requiere de diferentes procedimientos sintácticos que posibilitan la configuración de estructuras de diverso rango (grupos de palabras, cláusulas, complejos clausulares) con posiciones funcionales opuestas y solidarias entre sí. Por lo tanto, el manejo de una sintaxis compleja se evidencia cuando el sujeto es capaz de producir complejos clausulares que consideran diferentes grados de interdependencia y relaciones lógico-semánticas entre los elementos léxicos seleccionados.

Ahora bien, en el estudio de la complejidad sintáctica se han utilizado diferentes conceptos, tales como el de Unidad Mínima Terminal (Unidad T) y el de paquete clausular. La Unidad T, definida desde una perspectiva generativista, apunta a una estrutura sintácticamente autónoma compuesta por un verbo finito y sus complementos, que se analiza, por un lado, cuantitativamente considerando el promedio de palabras y cláusulas que la componen y, por otro, cualitativamente estableciendo las transformaciones realizadas (Crespo, Alfaro y Gongora, 2013). El paquete clausular, desde una perspectiva funcional-discursiva, corresponde a una unidad compuesta por varias cláusulas relacionadas sintáctica, temática y discursivamente, por lo cual cumplen diferentes roles a nivel local y global, como por ejemplo, introducir, desarrollar o sintetizar información. Al interior de un paquete clausular es posible identificar cinco tipos de relaciones, a saber, isotaxis (aislamiento), parataxis simétrica (yuxtaposición y cooordinación coherente semánticamente y con autonomía sintáctica), parataxis asimétrica (yuxtaposición y coordinación coherente semánticamente con cierta dependencia sintáctica), hipotaxis (subordinación a una cláusula subordinante), y endotaxis (incrustación y anidación dentro de otra cláusula) (Crespo, Alfaro y Gongora, 2013; Aravena y Hugo, 2016).

Por su parte, el concepto de complejo clausular, propuesto por la lingüística sistémicofuncional, posibilita no solo dar cuenta del grado de dependencia entre las clausulas identificando las relaciones paractácticas e hipotácticas que se dan en su interior, sino que aborda las relaciones lógico-semánticas de proyección y expansión entre ellas. En otras palabras, la relación lógicasemántica de proyección posibilita distinguir, al interior de un complejo clausular, si los fenómenos representados en la cláusula expresan ideas o reproducen el discurso de un sujeto. Las relaciones de expansión apuntan a analizar los procedimientos que se mantienen en un mismo orden de experiencia, ya sea elaborando, extendiendo o realzando la información. En el caso de la elaboración, una de las cláusulas especifica o describe una información previa; en la extensión, se agrega información nueva mediante procedimientos tales como, la denominación, la reformulación, el cambio de perspectiva; en el realce, se establecen relaciones circunstanciales (Halliday y Mathiessen, 1999, 2004; Thompson, 2014; Eggins, 2013).

Si bien las investigaciones realizadas han demostrado que un niño es capaz de producir oralmente complejos clausulares a temprana edad (4 años aproximadamente), su ingreso a la escuela potencia y fortalece el uso de este procedimiento debido a su interacción permanente con 


\section{Manuel Rubio}

diversos tipos de textos escritos. En efecto, en la etapa escolar se verifica un aumento en el número de unidades por oración, el mayor uso de estructuras poco frecuentes y el incremento de complejos clausulares (Coloma et al., 2007; Meneses et al., 2012; Ow y Alvarado, 2013; Crespo, Alvarado y Meneses, 2012; Aravena y Hugo, 2016).

A través de un estudio de recontado de tres cuentos infantiles, Coloma et al. (2007) constataron que los niños de 10 años producen más oraciones complejas que los niños de 8 años, por lo cual concluyeron que la complejidad sintáctica aumenta con la edad. Por su parte, Meneses et al. (2012) describieron la complejidad sintáctica en discursos monogestionados orales y escritos en producciones narrativas y explicativas de cuatro estudiantes de $5^{\circ}$ básico. Este estudio permitió establecer diferencias en el tipo de complejo clausular según el tipo de texto. En efecto, los textos narrativos presentan complejidad sintáctica interclausular (parataxis simétrica) y los explicativos complejidad sintáctica intraclausular (hipotaxis). Ow y Alvarado (2013) se enfocan en el estudio de la narración oral en estudiantes de cinco niveles escolares (kínder, $3^{\circ}$ básico, $6^{\circ}$ básico, $1^{\circ}$ medio y $4^{\circ}$ medio), lo cual les permitió concluir que, si bien los estudiantes utilizan relaciones hipotácticas a temprana edad, a medida que avanzan en la escolaridad se produce un incremento en la interdependencia asimétrica entre las clausulas, lo cual les permite plantear que la subordinación sustantiva posee un mayor grado de complejidad, pues aparece más tardíamente que ciertas subordinaciones adverbiales. Aravena y Hugo (2016) compararon los textos narrativos y explicativos escritos producidos por estudiantes de $7^{\circ}$ básico, $1^{\circ}$ medio y $3^{\circ}$ medio, de dos establecimientos escolares diferenciados de acuerdo con el nivel socioeconómico, concluyendo que los escolares de nivel socioeconómico alto construyen textos con una mayor complejidad sintáctica en cada uno de los cursos, evidenciando mayor elaboración y conexión de la información.

En síntesis, la escolarización desempeña un rol relevante en la elaboración de los recursos disponibles para la escritura de un texto. De hecho, diversos estudios constatan un incremento en el uso de complejos clausulares con relaciones asimétricas entre las cláusulas que los integran y comienza a aparecer con mayor fuerza relaciones semánticas de proyección, evidenciando el desarrollo de la capacidad para representar ideas y locuciones. Paralelamente, a medida que avanza la escolaridad, los escolares son capaces de usar la nominalización y, así, profundizar la densidad informativa del texto (Christie y Derewianka, 2010). En concreto, la escolarización introduce a los estudiantes en prácticas lingüísticas y culturales, en las cuales la escritura es tanto un objeto de estudio como una forma de comunicación y construcción de saberes (Schleppegrell, 2004).

\section{Metodología}

\section{Diseño}

La investigación corresponde a un estudio de carácter cuantitativo que pretende comparar los textos de estudiantes chilenos de tres niveles de escolaridad diferentes $\left(3^{\circ}, 5^{\circ}\right.$ y $7^{\circ}$ básicos $)$ con el propósito de identificar posibles cambios en el nivel clausular de las narraciones escritas producidas por ellos. Con este fin, se trabajó con estudiantes de un establecimiento particular subvencionado de la comuna de Santiago, correspondiente a un nivel socio-económico medio alto, según la clasificación entregada por el Ministerio de Educación. 


\section{Manuel Rubio}

\section{Participantes}

En el establecimiento seleccionado, había dos cursos por nivel, con 90 estudiantes por cada uno. De cada nivel, se seleccionó una muestra al azar de 30 estudiantes. El único requisito fue que hubieran completado las dos tareas de escritura. Para ello, se utilizó una tabla de número aleatorios.

\section{Tarea}

Se aplicaron dos tareas de escritura debidamente validadas: a) Una anécdota personal modelada, en la cual se describió y ejemplificó la tarea y se diseñó un dispositivo que permitiera que los escolares recordaran una experiencia vivida y b) el recontado luego de observar, en dos oportunidades y en silencio, una serie de imágenes. Estas tareas fueron validadas mediante el criterio de jueces expertos (Rubio, 2016).

\section{Consentimiento ético y procedimientos de aplicación}

En una reunión de apoderados, se explicó el objetivo y procedimientos de la investigación. Después de dicha explicación, se solicitó la firma de una carta de consentimiento informado del apoderado. Los apoderados que no estuvieron presente fueron citados por la profesora jefe, un procedimiento habitual en el establecimiento, instancia en el cual se les informó de la investigación y se solicitó su firma autorizando la participación de su familiar en el estudio. Cabe indicar que tanto el director del colegio como los profesores de lenguaje de cada curso firmaron una carta para expresar su consentimiento. En todos los casos, se aseguró la confidencialidad de los datos y su uso solo con fines académicos.

Para la narración de una experiencia vivida (anécdota personal modelada), se partió por la activación del conocimiento previo mediante un diálogo intencionado por el docente a cargo del curso sobre la labor de los escritores. Dicho diálogo se sistematizó de manera muy simple mediante la descripción de la labor de un escritor en relación con la reflexión sobre experiencias vividas. Además, se ejemplificó por medio de una anécdota sencilla con un escolar como protagonista y con la clara presencia de una complicación. Luego, se apeló al recuerdo lo más detallado posible de una vivencia personal libremente elegida por los escolares. Finalmente, se les planteó la tarea de jugar a ser escritores con la única exigencia de escribir para otras personas. Dicho procedimiento se especificó en un instructivo que fue explicado a los docentes que tuvieron la responsabilidad de aplicarlo en clases bajo la supervisión de un investigador (Rubio, 2016).

Para el recontado, se utilizaron las ilustraciones de un cuento álbum (El Tunel de Anthony Brown) debido a que los dos protagonistas están en edad escolar y son un hombre y una mujer, por lo cual se podría facilitar la identificación de los escolares con la situación descrita en dichas imágenes. Los escolares observaron las imágenes expuestas en un PowerPoint en dos oportunidades, ambas en silencio y tratando de imaginar un posible relato. La única exigencia de la tarea fue que sus textos iban a ser leídos por profesores y estudiantes de una universidad. Esta tarea también fue descrita en un instructivo explicado a cada docente, quienes lo aplicaron bajo la supervisión de un investigador (Rubio, 2016).

Cada uno de los 30 estudiantes por nivel, seleccionados en la muestra, escribió dos textos. En consecuencia, el corpus quedó conformado por un total de 180 textos, 90 por cada tarea de escritura, con las especificaciones que se indican en el cuadro $\mathrm{N}^{\circ} 1$. 
Manuel Rubio

Cuadro $\mathrm{N}^{\mathrm{o}} 1$. Extensión del corpus de análisis

\begin{tabular}{|l|c|c|c|c|}
\hline Curso & $\begin{array}{c}\text { Número total de } \\
\text { palabras tarea 1 } \\
\text { (anécdota pers. } \\
\text { modelada) }\end{array}$ & $\begin{array}{c}\text { Promedio de } \\
\text { palabras tarea 1 }\end{array}$ & $\begin{array}{c}\text { Número total de } \\
\text { palabras tarea 2 } \\
\text { (recontado) }\end{array}$ & $\begin{array}{c}\text { Promedio de } \\
\text { palabras tarea 2 }\end{array}$ \\
\hline 3o básico & 3328 & 110 & 3379 & 113 \\
\hline 5o básico & 3983 & 133 & 4979 & 166 \\
\hline 70 basico & 5774 & 193 & 5933 & 198 \\
\hline Total & 13085 & 145 & 14291 & 159 \\
\hline
\end{tabular}

\section{Segmentación y codificación}

Cada texto se segmentó en cláusulas simples y complejos clausulares, para lo cual se consideraron los verbos explícitamente expresados en el texto y que estuvieran conjugados, como se ejemplifica a continuación:

[1( $\alpha)]$ Había una vez un niño ( $\beta$ que jugaba futbol y (2) un día se fue a probar a cobreloa cadete

(1) estaba 3 dias probandose [2( $\alpha)$ ]y al cuarto dia le dijieron ( $\beta$ )que no iva a quedar en el club

(1) el se frustro (2) y no jugo 1 mes en ningun equipo de futbol

$[1(\alpha)]$ un dia miercoles del mes de marzo se inscribe en un club $(\beta)$ que se llamaba sporting

(1) al primero partido por ese club se hizo un gol al segundo partido un golazo (2)y al tercero jugo mas o menos

$[1(\alpha)]$ y se fustro mucho $(\beta) x q$ se perdio muchos goles.

(1) ahora espera todos los sabados para ir a jugar.

Los números indican las relaciones paratácticas entre las clásulas y las letras griegas las relaciones hipotácticas. En este caso, la $\alpha$ indica la cláusula dominante y la letra $\beta$ la clásula dependiente. Posteriormente, se etiquetaron las relaciones lógico-semánticas de expansión y proyección. Se aplicó un procedimiento de doble codificación por dos estudiantes de magíster en lingüística que fueron previamente entrenados para hacerlo.

\section{Plan de análisis}

Se determinó la frecuencia de cláusulas simples y complejos clausulares por cada texto. Asimismo, se calculó la frecuencia de relaciones paratácticas e hipotácticas y la frecuencia en el tipo de 
Manuel Rubio

relación lógico-semántica presente en los complejos clausulares (expansión y proyección). Los datos de cada texto se exportaron a una planilla excell y se configuraron gráficos que muestran las diferencias entre los cursos. Para identificar si las diferencias entre niveles escolares eran significativas, se utilizó como estadígrafo el chi-cuadrado ( $p<0.05$; Levon, 2010; López-Roldán y Fachelli, 2015).

\section{Resultados}

Un primer aspecto analizado es la frecuencia y el promedio de cláusulas simples y de complejos clausulares en cada texto escrito por los estudiantes. En ambas tareas de escritura, predominan los complejos clausulares, como se observa en el gráfico 1.

Gráfico 1. Frecuencia de cláusulas simples y complejos clausulares.

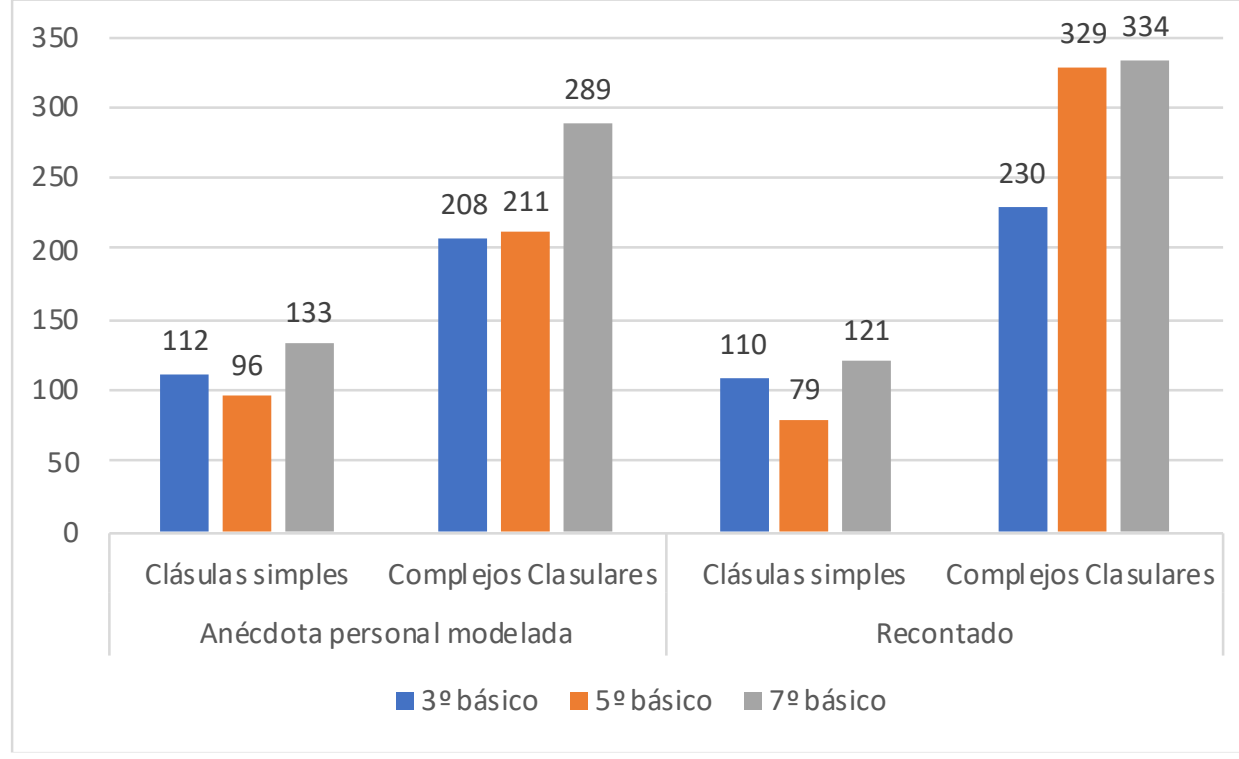

En el gráfico 2, se puede observar las diferencias en el incremento de complejos clausulares en las dos tareas de escritura. En la anécdota personal modelada, la mayor diferencia se da entre el $5^{\circ}$ y el $7^{\circ}$ básicos; en el recontado, el mayor incremento se registra entre el $3^{\circ}$ y el $5^{\circ}$ básico.

Gráfico 2. Incremento en la frecuencia de complejos clausulares

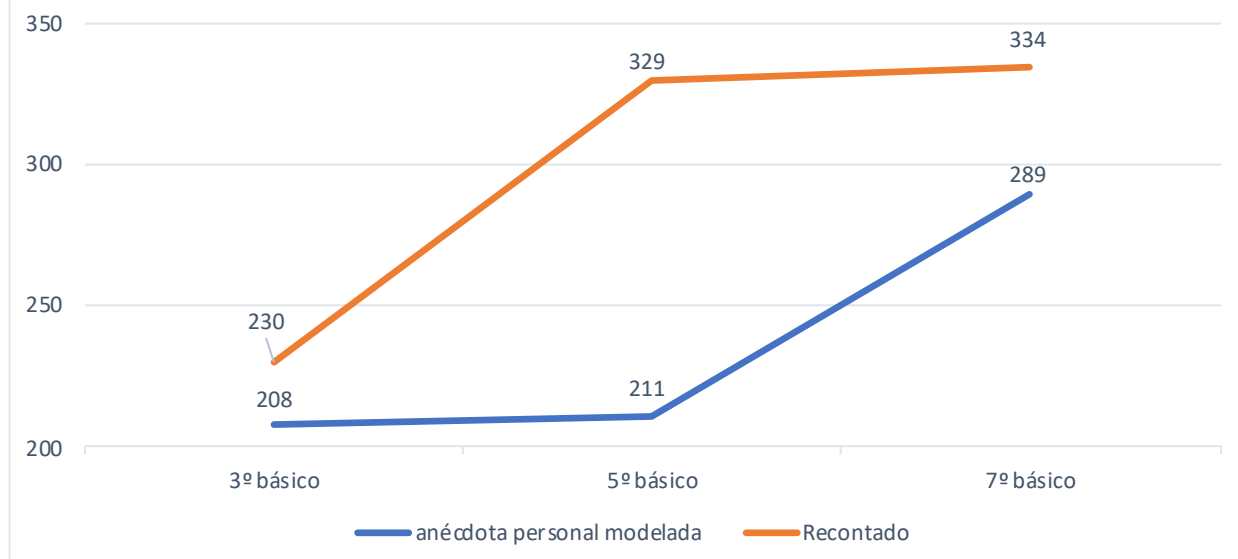




\section{Manuel Rubio}

Respecto de la influencia del factor nivel escolar en la proporción de cláusulas simples y complejos clausulares presentes en las narraciones escritas por los estudiantes, se observa que en la anécdota personal modelada el factor nivel escolar no es significativo ( $p>0,5197)$ mientras que sí lo es en el recontado ( $\mathrm{p}<0,00025)$. En el recontado, se registra una mayor frecuencia de complejos clausulares. Al parecer, la configuración previa de la globalidad del relato facilitado por la observación de imágenes, contribuyó a precisar la relación de dependencia de los eventos narrados y las relaciones lógico semántica entre ellos. Las imágenes como estímulo previo a la producción de la narración escrita habría brindado la posibilidad de construir una representación mental global de lo ocurrido, identificando con mayor claridad la situación inicial, la acción desencadente que generó el desequilibrio y la resolución final.

\section{Grado de dependencia entre clásulas (hipotaxis y parataxis)}

Al analizar los complejos clausulares de acuerdo con el grado de dependencia de las cláusulas, se constata que en los textos escritos por escolares predomina la parataxis, es decir, secuencias de cláusulas que se conjuntan, en las cuales una inicia un evento y las otras lo complementan en términos de reformulación, agregación de elementos o explicitación de rasgos circunstanciales (gráfico 3).

Gráfico 3. Grado de dependencia de cláusulas expresado en frecuencia.

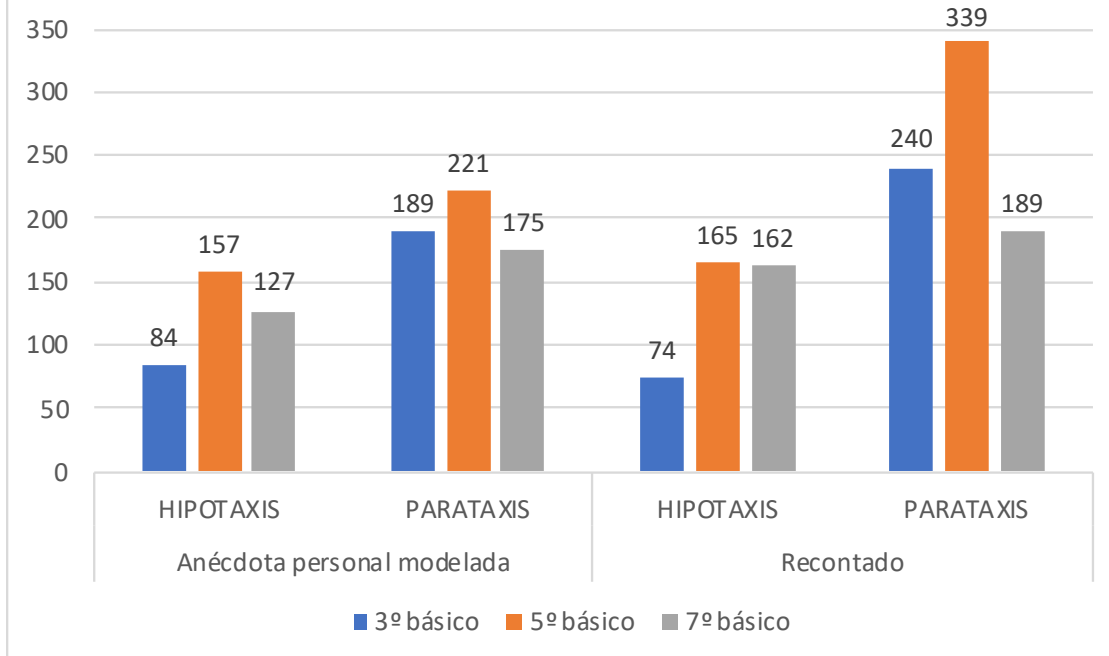

Cuando se analiza la influencia de la variable nivel escolar sobre el grado de dependencia, se constata que hay una diferencia significativa en la proporción de complejos clausulares con dependencia hipotáctica y paratáctica $(\mathrm{p}<0,0412$ para la anécdota personal modelada y $\mathrm{p}<0,0000$ para el recontado). Las relaciones paratácticas en los complejos cláusulares presenta un incremento entre el $3^{\circ}$ y el $5^{\circ}$ en ambas tareas de escritura, pero tiende a disminuir en el $7^{\circ}$ básico. Por el contrario, las relaciones hipotácticas presentan un patrón de incremento diferente: existe un aumento relevante entre el $3^{\circ}$ y el $5^{\circ}$ básicos, el cual tiende a mantenerse en el $7^{\circ}$ básico más en el recontado que en la anécdota personal. Al parecer, la hipotaxis sería un grado de dependencia más complejo para los escolares. Dicho incremento se evidencia en el gráfico 4. 


\section{Manuel Rubio}

Gráfico 4. Frecuencia de complejos clausulares con dependencia hipotáctica.

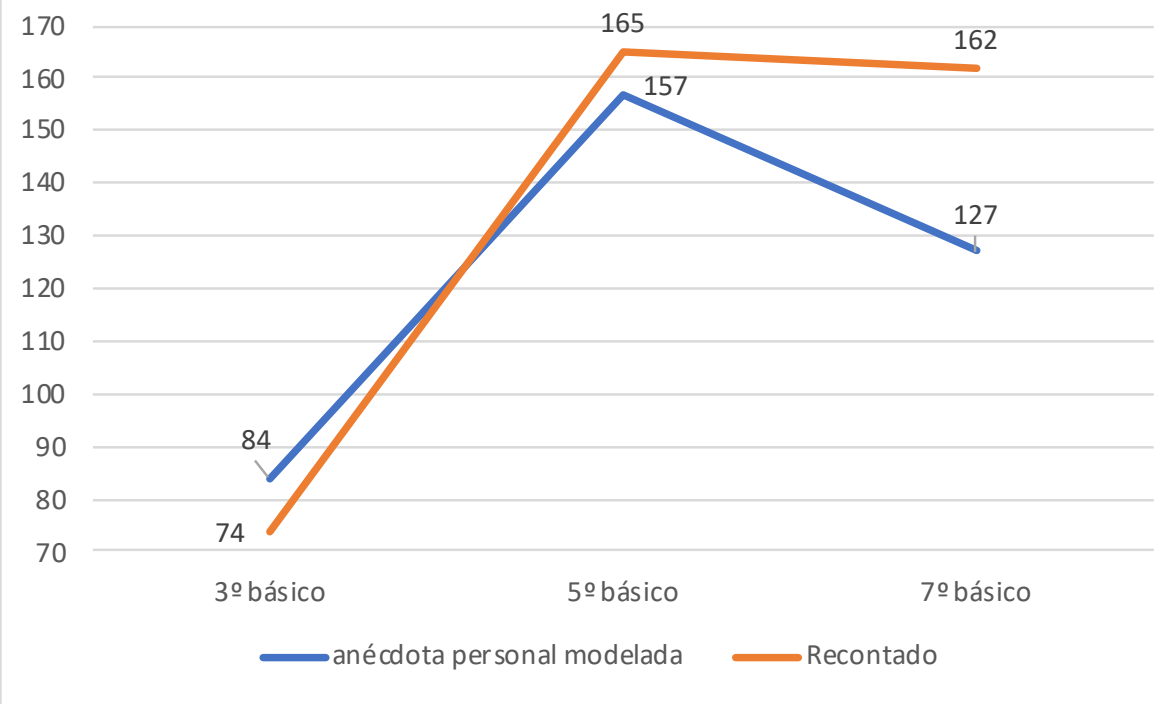

\section{Relaciones lógico-semánticas entre clásulas (proyección y expansión)}

Al considerar la relación lógica semántica en los textos escritos por los escolares, se aprecia un predominio de la expansión por sobre la proyección, lo cual indica que los escolares tienden a complejizar sus textos mediante procedimientos orientados a enriquecer la descripción de los eventos narrados más que a expresar el pensamiento o el habla de los personajes representados. En otras palabras, privilegian un tipo de narrador que caracteriza externamente los eventos, centrando su atención en la acción representada más que en la interioridad de los sujetos que la realizan. El gráfico 5 muestra las relaciones lógico-semánticas contenidas en los complejos clausulares de los escolares.

Gráfico 5: Frecuencia de relaciones lógico-semánticas

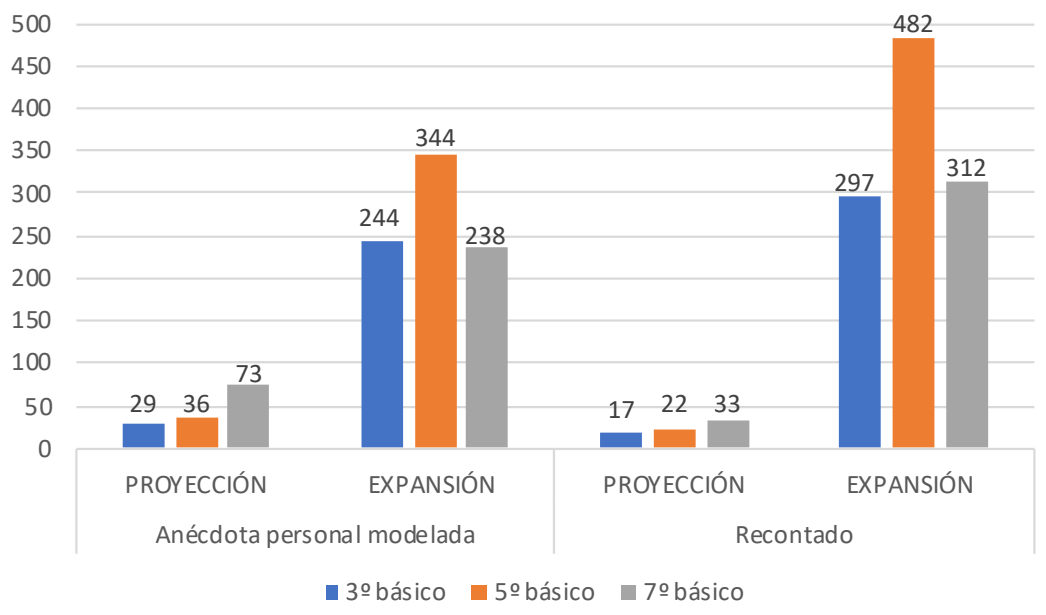

Cuando se analiza la asociación entre la variable nivel escolar y las relaciones lógicosemánticas entre cláusulas, se constata que hay una diferencia significativa en la proporción de complejos clausulares con relación de expansión ( $\mathrm{p}<0,0000$ para la anécdota personal modelada y 


\section{Manuel Rubio}

$p<0,0068$ para el recontado). Este incremento se da en todos los niveles escolares, especialmente en el $5^{\circ}$ año para ambos tipos de tarea. Al tratar de identificar los procedimientos semánticos utilizados por los escolares para dotar a los eventos representados en sus textos con un mayor nivel de detalles, considerando solo los complejos clausulares con una relación de expansión, se constata que en ambos textos:

a) predominan los recursos orientados a darle mayor realce al evento representado en una cláusula con otra que destaca elementos de carácter circunstancial que especifican rasgos temporales, espaciales, causales o de condición.

b) le siguen los recursos cuyo propósito es extender la representación de los eventos mediante una cláusula que agrega información nueva mediante elementos conjuntivos.

c) En último lugar los escolares utilizan recursos destinados a elaborar el significado de una cláusula o de algún elemento de ella, sin necesariamente aportar nueva información, sino que profundizando lo previamente expuesto. En otras palabras, tienden a no reelaborar el significado de un elemento previamente representado mediante procedimientos de aclaración, ejemplificación, redefinición, caracterización o explicación.

Finalmente, cabe indicar que la elaboración paratáctica tiende a privilegiar, en ambos tipos de textos, la realización marcada por un nexo conjuntivo. En el caso de la hipotaxis, se tiende a privilegiar la elaboración finita en $3^{\circ}$ y $5^{\circ}$ años, circunscribiendo temporal y modalmente la cláusula dependiente introducida por un relativo, y la elaboración no finita en $7^{\circ}$ año.

En resumen, pareciera que la complejización mediante el realce de elementos circunstanciales es la más fácil de expresar por los estudiantes. En tanto que los elementos destinados a la extensión siguen un patrón de relativo incremento al avanzar en los niveles de escolaridad, es decir, los textos se harían más informativos mediante la adición de nuevos elementos, sin que ello signifique necesariamente que haya una mayor elaboración de los mismos.

Si bien las cláusulas que expresan relaciones lógico semánticas de proyección en los complejos clausulares son pocas (ver gráfico 5), pues los estudiantes prácticamente no se detienen en destacar el pensamiento o el habla de los actores representados, es importante señalar que en ellas claramente predomina la representación del discurso de los sujetos representados. No obstante, tiende a incrementarse la representación de las ideas mediante el uso de verbos de carácter mental a medida que se avanza en la escolaridad, especialmente en el último nivel analizado, como se evidencia en el gráfico 6. 


\section{Manuel Rubio}

Gráfico 6: Porcentaje del tipo de proyección en los complejos clausulares

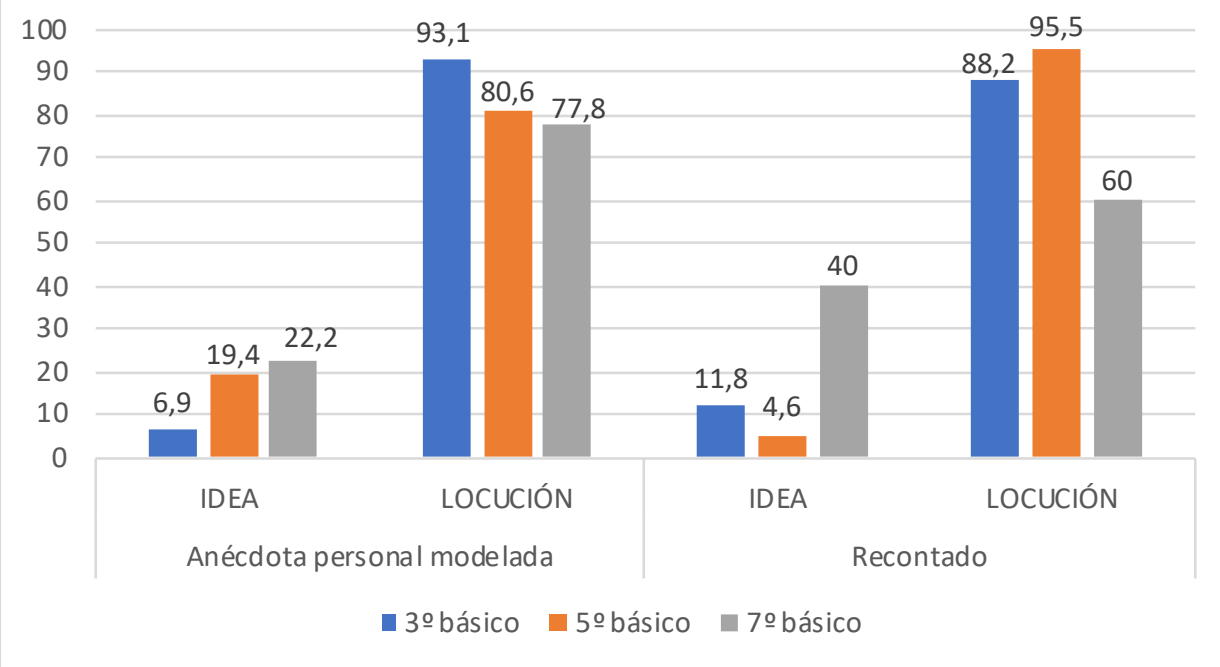

En la anécdota personal modelada, se aprecia un incremento sostenido de complejos clausulares con proyección de ideas. En el recontado, se puede observar un importante aumento de complejos clausulares con proyección de ideas ente el $5^{\circ}$ y el $7^{\circ}$ básicos. En otras palabras, es posible deducir que el avance en la escolaridad supone mayores recursos para construir un relato escrito que manifieste la reflexión sobre los eventos narrados, concretando dicho proceso mental como un proceso del narrador o de algunos de los personajes representados.

\section{Conclusiones}

En contraste con el estudio del desempeño oral, en español existen pocos estudios orientados al análisis lingüístico de textos escritos por escolares de Educación Básica. En general, se ha analizado poco el efecto de la variable nivel escolar en el uso de recursos lingüísticos en la escritura. La descripción del análisis clausular aplicado a las narraciones escritas por estudiantes de tres niveles de escolaridad indica que dicha variable impacta en el incremento de complejos clausulares. Si bien en los textos analizados predominan significativamente los complejos clausulares por sobre la cláusula simple, dicho predominio se incrementa significativamente entre el $3^{\circ}$ y el $5^{\circ}$ año básico y tiende a estabilizarse en el $7^{\circ}$ básico. Este resultado es congruente con los estudios que evidencian un cambio significativo en el tipo de verbos utilizados en sus textos escritos por los escolares (Rubio, 2019) y en los elementos de conexión textual (Díaz Oyarce (2019).

Un patrón de incremento en los complejos clausulares por sobre las cláusulas simples indica un mayor nivel de complejidad de los textos escritos por los escolares, puesto que implica integrar en una misma estructura sintagmática una secuencia de procesos encadenados entre sí, explicitando su jerarquía y relación lógico-semántica. Dicho incremento se facilitaría por las prácticas letradas que se dan en la escuela. De hecho, se ha demostrado que dichas prácticas inciden en la complejidad sintáctica de relatos orales (Coloma et al., 2007; Meneses et al., 2012; Ow y Alvarado, 2013; Crespo et al., 2012) y en los escritos (Aravena y Hugo, 2016). Incluso, habría un uso estratégico de recursos sintácticos complejos para el logro de fines comunicativos (Crespo et al., 2012; Schleppegrell, 2004; Christie y Derewianka, 2010).

Si bien en los textos analizados predomina la parataxis, se aprecia un descenso de esta a medida que se avanza en la escolaridad. En cambio, las relaciones de hipotaxis tienden a presentar 


\section{Manuel Rubio}

un patrón de incremento que logra niveles significativos al comparar el $3^{\circ}$ con el $5^{\circ}$ básico y un avance más gradual al comparar este último nivel con el $7^{\circ}$ básico. De algún modo, esto significa que los estudiantes progresivamente avanzan en la condensación de información en una misma estructura sintagmática, estableciendo un proceso dominante representado por un verbo conjugado y procesos dependientes representados ya sea por verbos conjugados o no conjugados. Esto es coherente con lo planteado por Ow y Alvarado (2013), quienes sostienen que, en el plano oral, el avance en la escolaridad impacta en el uso de relaciones hipotácticas en la producción textual de los escolares. Al respecto, Christie y Derewianka (2010) indican que, entre los 9 y 12 años aproximadamente, los niños viven una etapa de su desarrollo lingüístico caracterizado por un mayor despliegue de recursos léxico-gramaticales para configurar la experiencia, las relaciones lógicas entre los eventos, las relaciones interpersonales y la progresión temática. En este sentido, señalan que este sería un periodo de transición hacia formas de uso de la lengua cercanas a un registro característico de la escritura académica.

La relación lógico-semántica predominante característica de los complejos clausulares en los textos analizados es la de expansión, lo cual indica que los estudiantes aplican una serie de recursos para enriquecer la descripción de los eventos representados. En otras palabras, una cláusula se conecta semánticamente con otra, ya sea para destacar elementos circunstanciales que configuran más claramente el escenario en que un proceso ocurre o para agregar información nueva mediante elementos conjuntivos. En este sentido, se utilizan pocos recursos orientados a elaborar lo previamente expuesto, sea a través de operaciones de reformulación, aclaración, ejemplificación, redefinición o explicación.

Los complejos clausulares analizados presentan pocas relaciones lógico-semánticas de proyección del habla o el pensamiento. No obstante, se puede visualizar un avance gradual en el uso de recursos lingüísticos para poner de manifiesto la reflexión del narrador o los participantes en los eventos narrados. Esto es concordante con lo descrito por Rubio (2019), que indica que con la escolaridad hay un incremento gradual en el uso de cláusulas que expresan procesos verbales y mentales, aunque es significativo el predominio, en los escritos escolares, de cláusulas que representan procesos materiales, es decir, que los escolares tienden a representar la experiencia como un evento externo, lo que gradualmente va cambiando con la escolaridad al incorporar el uso de verbos que sitúan la experiencia desde la subjetividad del narrador. Esto es interesante, puesto que, según ha constatado Bocaz (1996), los niños antes del ingreso a la escuela (a los 5 años aproximadamente) están en condiciones de producir relatos orales que combinen el paisaje de la acción con el paisaje de la conciencia, es decir, son capaces de situarse en la interioridad de los actantes y atribuirles intenciones. Al parecer, un obstáculo para hacer esto en el plano escrito estaría dado por la complejidad que implica esta modalidad del uso de la lengua.

En definitiva, es posible postular que en el $5^{\circ}$ año se produce un cambio cualitativo en las narraciones escritas de los estudiantes quienes producen un mayor número de complejos clausulares en los que predominan las relaciones hipotácticas, las cuales condensan y jerarquizan una mayor cantidad de procesos, estableciendo relaciones semánticas más precisas entre ellos. Dicho cambio se favorece cuando los estudiantes pueden previamente contar con estímulos previos que posibiliten la configuración global del conjunto de episodios que integrarán su texto.

Los estudiantes en los tres niveles de escolaridad analizados producen textos detallando los eventos representados mediante recursos lingüísticos que destacan las circunstancias y agregan información nueva, sin profundizar mayormente en lo ya dicho mediante el uso de ejemplos, aclaraciones o redefiniciones, entre otros.

En general, los estudiantes escriben narraciones que relevan las acciones como eventos observables, cuyos participantes prácticamente no hablan ni reflexionan sobre su quehacer. No

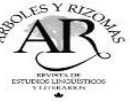




\section{Manuel Rubio}

obstante, hay una tendencia incipiente a enriquecer sus producciones incorporando estos aspectos a medida que se avanza en la escolaridad.

En consideración de estas conclusiones, resulta importante analizar textos de estudiantes de otros niveles escolares para establecer el curso de los cambios, contando con distinciones que permitan caracterizar más precisamente la evolución de los grados de interdependencia y las relaciones lógico-semánticas en los complejos clausulares en sus textos escritos. De este modo, se contará con un cuerpo de conocimiento para describir el horizonte de posibilidades que tienen los escolares de cada nivel cuando escriben y, así, formular estrategias pedagógicas pertinentes y potenciadoras de su capacidad de construir significados. Cabe hacer notar que, dado que la cultura letrada de la familia es relevante en la complejización de los textos (Aravena y Hugo, 2016), es necesario configurar dispositivos pedagógicos que pongan en relación a los escolares con diversos géneros discursivos para explicitar las relaciones entre las cláusulas y orientar el proceso de producción textual.

En términos de las limitaciones del estudio, se debe precisar que este se hizo con escolares de un mismo nivel socioeconómico. Por tanto, para caracterizar más precisamente el incremento de diferentes recursos lingüísticos utilizados en la producción escrita por los escolares básicos, es conveniente comparar textos producidos por estudiantes de diferentes sectores sociales con el fin de constatar si los patrones descritos siguen la misma tendencia. Al mismo tiempo, sería importante controlar en el corpus la presencia de textos cuya extensión escapa de la norma. Dicha normalización contribuiría a precisar los patrones de incremento de los complejos clausulares.

\section{Referencias}

Aravena, S. (2011). El desarrollo narrativo a través de la adolescencia: estructura global de contenido y referencia personal. Signos 44 (77), 215-232. https://doi.org/10.4067/s0718-09342011000300002

Aravena, S. y Hugo, E. (2016). Desarrollo de la complejidad sintáctica en textos narrativos y explicativos escritos por estudiantes secundarios. Lenguas modernas 47, 9-40.

Bañales, G., Ahumada, S., Martínez, R., Martínez, M. y Messina, P. (2018). Investigaciones de la escritura en la Educación Básica en Chile: revisión de una década (2007-2016). RLA 56(1), 59-84. https://doi.org/10.4067/s0718-48832018000100059

Beard, R., Myhill, D., Riley, J. and Nystrand, M. (2009). The Sage handbook of writing development. London: Sage.

Benitez, R. (2009). Análisis descriptivo de narraciones escritas por niños y niñas de tercer año básico. Literatura y Lingüística 20, 103-123. https://doi.org/10.29344/0717621x.20.1565

Bocaz, A. (1996). El paisaje de la conciencia en la producción de narraciones infantiles. Lenguas modernas 23, 49-70.

Christie, F. y Derewianka, B. (2010). School Discourse. London: Continuum.

Coloma, C. J., Peñaloza, C. y Reyes Fernández. (2007). Producción de oraciones complejas en niños de 8 y 10 años. RLA 45(1), 33-44. https://doi.org/10.4067/s0718-48832007000100003

Crespo, N., Alfaro, P. y Gongora. B. (2013). La medición de la sintaxis: evolución de un concepto. Onomázein 24(2), 155-172. 


\section{Manuel Rubio}

Crespo, N., Alvarado, C. y Meneses, A. (2012). Desarrollo sintáctico: una medición a partir de la diversidad clausular. Logos 23(1), 80-101.

Díaz Oyarce, C. (2019). La presencia de elementos de conexión en las producciones narrativas escritas en una muestra de escolares de Santiago de Chile. Signos 52(101), 736-758. https://doi.org/10.4067/s0718-09342019000300736

Eggins, S. (2013). An introduction to systemic functional linguistics. London: Bloomsbury.

Grabe, W. and Kaplan, R. (2014). Theory and practice of writing. New York: Longman.

Gutiérrez, R. M. (2008). El género manual en las disciplinas académicas: una caracterización desde el sistema de la obligación. Signos 41(67), 177-202. https://doi.org/10.4067/s0718-09342008000200007

Halliday, M.A.K. and Matthiessen, C. (1999). Construing experience through meaning: $A$ language-based approach to cognition. London: Cassell.

Halliday, M.A.K. and Matthiessen, C. (2004). An introduction to functional grammar. London: Arnold.

Kellog, R. (2008). Training writing skill: a cognitive development perspective. Journal of Writing Research 1 (1), 1-26.

DOI: $10.17239 /$ jowr-2008.01.01.1

Koster, M., Tribushinina, E., de Jong, P. and van den Bergh, H. (2015). Teaching children to write: a meta-analysis of writing intervention research. Journal of Writing Research 7 (2), 299324.

https://doi.org/10.17239/jowr-2015.07.02.2

Levon, E. (2010). Organizing and processing your data. The nuts and bolts of quantitative analyses. En L. Litosseliti (ed.), Research Methods in Linguistics (pp.68-92). London: Continuum.

López-Roldan, P. y Fachelli, S. (2015). Metodología de la investigación social cuantiativa. Barcelona: Universitat Autonóma de Barcelona.

Marinkovic, J. (2006). La escritura como proceso. Fundamentos y prácticas en el aula. Santiago: Frasis.

Martin, J., Mathiessen, C. and Painter, C. (1997). Working with functional grammar. London: Arnold.

Meneses, A. (2006). Leer y escribir en la escuela: representaciones discursivas de los diferentes agentes educativos en las áreas prioritarias del currículo escolar en NB3. Tesis para optar al grado de doctor en lingüística. Valparaíso: PUCV. https://doi.org/10.4067/s0718-09342008000200010

Meneses, A. (2008). Leer y escribir en una escuela chilena: representaciones discursivas de los diferentes agentes educativos en las áreas prioritarias del currículo escolar de NB3. Signos 41 (67), 257-278.

https://doi.org/10.4067/s0718-09342008000200010

Meneses, A., Ow, M. y Benitez, R. (2012). Complejidad sintáctica: ¿modalidad comunicativa o tipo textual? Estudio de casos de producciones textuales de estudiantes de $5^{\circ}$ básico. Onomázein 25, 65-93.

Mineduc (2009). Informe de resultados de escritura $4^{o}$ básico. Santiago: Mineduc.

Mineduc (2015). Informe de resultados de escritura $6^{\circ}$ básico. Santiago: Mineduc.

Ow, M. y Alvarado, C. (2013). Niños que narran: aumento en la complejidad discursive y sintáctica durante la edad escolar. Literatura y Lingüística 28, 149-168.

https://doi.org/10.29344/0717621x.28.71 
Manuel Rubio

Pinheiro, F. e de Freitas Rossi, T. (2008). A consciência metalingüística pragmática e sua relação com a produção escrita. RLA Revista de Lingüística Teórica y Aplicada 46(2), 29-51. https://doi.org/10.4067/s0718-48832008000200003

Rose, D. and Acevedo, C. (2017). Learning to write, Reading to Learn: background and development of genre-based literacy improvement projects in Australia. Lenguaje y textos 46, 7-18.

DOI: $10.4995 / \mathrm{lyt} .2017 .8688$

Rose, D. and Martin, J. (2012). Learning to write, Reading to learn. Genre, knowledge and Pedagogy in the Sydney School. South Yorkshire: Equinox.

Rubio, M. (2016). Narraciones de escolares básicos: dos tareas de escritura. Literatura y Lingüística 34, 221-249. https://doi.org/10.4067/s0716-58112016000200011

Rubio, M. (2019). Procesos experienciales en narraciones sobre situaciones cotidianas escritas por escolares básicos. Signos 52(99), 158-180. https://doi.org/10.4067/s0718-09342019000100158

Schleppegrell, M. (2004). The language os schooling. A Functional linguistic perspective. New Jersey: Lawrence Erlbaum Associates.

Sotomayor, C., Lucchini, G., Bedwell, P., Biedma, M., Hernández, C. y Molina, D. (2013). Producción escrita en la Educación Básica: análisis de narraciones de alumnos de escuelas municipales de Chile. Onomázein 1(27), 53-77. https://doi.org/10.4067/s0718-09342013000100005

Tomasello, M. (2003). Constructing a language. A usage-based language theory language acquisition. Massachusetts: Harvard University Press.

Thompson, G. (2014). Introducing functional. Grammar. London: Routledge 